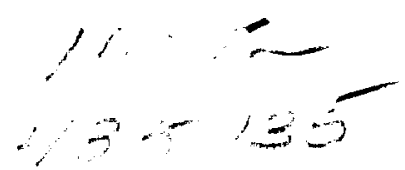

\title{
QUIET-TIME SPECTRA AND ABUNDANCES OF ENERGETIC PARTICLES DURING THE 1996 SOLAR MINIMUM
}

\author{
Donald V. Reames \\ NASA/Goddard Space Flight Center, Greenbelt, MD 20771 \\ reames@lheavx.gsfc.nasa.gov
}

Accepted for publication in Astrophysical Journal 


\begin{abstract}
We report the energy spectra and abundances of ions with atomic number, $Z$, in the interval $2 \leq \mathrm{Z} \leq 36$ and energies $-3-20 \mathrm{MeV} / \mathrm{amu}$ for solar and interplanetary quiet periods between 1994 November and 1998 April as measured by the large-geometry LEMT telescope on the Wind spacecraft near Earth. The energy spectra show the presence of galactic (GCR) and "anomalous" cosmic ray (ACR) components, depending on the element. ACR components are reported for $\mathrm{Mg}$ and $\mathrm{Si}$ for the first time at $1 \mathrm{AU}$ and the previous observation of $\mathrm{S}$ and $\mathrm{Ar}$ is confirmed. However, only GCR components are clearly apparent for the elements $\mathrm{Ca}, \mathrm{Ti}, \mathrm{Cr}, \mathrm{Fe}$, as well as for $\mathrm{C}$. New limits are placed on a possible ACR contribution for other elements, including $\mathrm{Kr}$.
\end{abstract}

Subject headings: acceleration of particles - shock waves - cosmic rays: abundances interstellar matter: abundances 


\section{INTRODUCTION}

As the Sun becomes less active during solar minimum, the number and size of both impulsive and gradual solar energetic particle (SEP) events are reduced and other sources of accelerated particles become more visible in the inner heliosphere. During the 1996 solar minimum there also appears to be a substantial reduction in the number of particles accelerated at corotating interaction regions (CIRs) where high- and low-speed solar wind streams collide to form shock waves. These quiet conditions are ideal for observing of rare elements accelerated at continuous heliospheric sources, such as the anomalous cosmic rays (ACRs), with the new instrument on Wind spacecraft that has a sensitivity 100 times greater than instruments that were available during the previous solar minima.

ACRs were first observed as increases in the intensities of $\mathrm{N}$ and $\mathrm{O}$ near $\sim 10$ MeV/amu that were not accompanied by an increase in $\mathrm{C}$ so that $\mathrm{O} / \mathrm{C}>20$ and $\mathrm{He} / \mathrm{O} \sim 1$ (e.g. McDonald et al. 1974). This O/C ratio, subsequently found to be $>50$ below 10 MeV/amu (Mewaldt et al. 1996), is truly anomalous in comparison to all other known heliospheric and GCR sources where O/C is between 1 and 2.5 (e.g. Meyer 1985, 1989; Reames 1995; Lund 1989). The early observers coined the name "anomalous cosmic rays" for this quiet-time particle population with its anomalous abundances and spectra before the origin of the population was known. ACR observations in the inner and outer heliosphere have been reviewed recently by Klecker (1995) and by Cummings and Stone (1996), respectively.

Fisk, Kozlovsky, \& Ramaty (1974) advanced the explanation for the source of the ACR He, N, O, and $\mathrm{Ne}$ that still persists today. Elements with a first ionization potential (FIP) greater than that of $\mathrm{H}$ are neutral in the interstellar medium just outside the heliosphere while those with lower FIP are ionized. The neutrals freely enter the heliosphere while the ions are excluded by the magnetic fields convected outward by the solar wind. As the neutrals approach the Sun, they can be photo-ionized, or charge exchange with the solar wind, so they are suddenly able to interact with the magnetic field and are "picked up" by the solar wind that carries that field. The pick-up ions have a velocity distribution function that extends from zero to twice the solar wind speed. The pick-up ions are eventually convected out to the heliospheric termination shock. Here, we now believe, they are preferentially accelerated because they have a higher average injection speed than the ions of the ambient solar wind plasma (Pesses, Jokipii \& Eichler 1981; Lee 1996; Fisk 1996). Ions with higher speed are better able to overtake the quasiperpendicular termination shock before being swept downstream. Finally, the accelerated ACR ions are modulated as they propagate back into the inner heliosphere against the flow of the solar wind. However, the modulation of the ACRs is less severe than that of the GCRs because the ACRs are singly ionized and have much higher magnetic rigidity than the fully ionized GCRs at the same speed. The theory of Fisk, Kozlovsky, \& Ramaty (1974) not only explained the observation of ACRs, but it also predicted the existence of pick-up ions in the solar wind many years before they were observed directly.

Using the geomagnetic field to separate ionization states, Adams et al. (1991) showed the first conclusive evidence that ACR $O$ was indeed singly ionized. In recent years, observations on the Solar, Anomalous and Magnetospheric Particle Explorer (SAMPEX) have used the same technique to confirm the ionization state of ACR ions (see Klecker 1995 and references therein). Most of the ions are indeed singly charged, 
although stripping during acceleration does produce a small component of multiply ionized ions that increases in importance with increasing energy (Mewaldt et al. 1996; Klecker et al. 1998). In the solar wind itself. Möbius et al (1985) made the first direct observation of $\mathrm{He}^{+}$pickup ions, but pickup $\mathrm{H}^{+}, \mathrm{O}^{+}, \mathrm{N}^{+}$, and $\mathrm{Ne}^{+}$were not observed until more recently (Gloeckler et al. 1993; Geiss et al. 1994).

During the recent solar minimum, the ACR elements Ar and S were observed at 1 $\mathrm{AU}$ for the first time (Hasebe et al. 1997; Reames, Barbier, \& von Rosenvinge 1997a; Takashima et al. 1997). Following these new observations at $1 \mathrm{AU}, \mathrm{S}$, as well as Si and $\mathrm{Fe}$, have been reported from Voyager observations (Stone \& Cummings 1997).

The present paper reports the results of observations by the large-geometry telescope on Wind of element spectra and abundances in the $-3-20 \mathrm{MeV} / \mathrm{amu}$ region during the full 3.5-year time period surrounding solar minimum. Preliminary results from the first 1.5 years of observation were reported by Reames et al. (1997a).

\section{DATA SELECTION AND RESOLUTION}

All new observations reported in this paper were made with the Low Energy Matrix Telescope (LEMT) of the Energetic Particles, Acceleration, Composition and Transport (EPACT) experiment on the Wind spacecraft. Von Rosenvinge et al. (1995) described this instrument in considerable detail. LEMT achieves a geometry factor of $51 \mathrm{~cm}^{2} \mathrm{sr}$ using three individual telescopes, each with a 16-element domed array of 17-micron-thick Si detectors followed by a large position-sensing detector (PSD) $1 \mathrm{~mm}$ thick and an anticoincidence element. LEMT is capable of measuring all chemical elements from $\mathrm{H}$ through $\mathrm{U}$, with varying element resolution, in an energy interval of approximately 2-20 $\mathrm{MeV} / \mathrm{amu}$, depending upon the species. In this paper we will limit our study primarily to the 3.3-20 MeV/amu region where all species under consideration are detected and are reasonably well resolved. The LEMT geometry factor is $\sim 100$ times larger than instruments covering these energies during previous solar minima. During the current epoch, LEMT is the only large-geometry instrument capable of measuring below $\sim 10 \mathrm{MeV} / \mathrm{amu}$. Thus, it is uniquely suited to investigate the possible existence of low-intensity ACR components such as $\mathrm{Mg}, \mathrm{Si}, \mathrm{S}, \mathrm{Ar}, \mathrm{Ca}$, and $\mathrm{Fe}$ in the energy region where they are expected.

Quiet-time periods were primarily defined to be 8-hour periods during which intensities of 2.1-2.4 MeV protons were below $100\left(\mathrm{~m}^{2} \mathrm{sr} \mathrm{s} \mathrm{MeV}\right)^{-1}$ as in previous studies (e.g. Richardson et al. 1990). However, we also required that the intensities of $2-4 \mathrm{MeV} / \mathrm{amu}$ $\mathrm{He}$ ions be below $0.3\left(\mathrm{~m}^{2} \mathrm{sr} \mathrm{s} \mathrm{MeV/amu}\right)^{-1}$. The latter criterion was determined by plotting a distribution of the frequency of occurrence of 8-hour averaged intensities of He; the criterion was placed above the large peak representing the persistent quiet-time ACR He. This harsh criterion was deemed necessary to effectively eliminate the low intensities of ions from CIRs that have recently been observed (Reames et al. 1997c). In addition, times of impulsive and gradual SEP events were explicitly excluded, including a time several hours prior to the apparent particle onsets when "velocity dispersion" might allow unseen high-energy particles to be present (see Reames et al. 1997b) before the low-energy $\mathrm{H}$ and $\mathrm{He}$ intensities increase. 
The above criteria yield a total quiet time of 1.6 years out of the 3.5 years, most of it during 1996 and early 1997. The percentage of time that we find to be quiet is plotted in Figure 1 together with the intensity of anomalous $O$ at several energies averaged over 27 day intervals. For much of the period the $O$ intensity was fairly flat above $8 \mathrm{MeV} / \mathrm{amu}$ but gently rising with time at lower energies. The onset of the new solar cycle began in late 1997 and early 1998 with an increasing number of both impulsive and gradual SEP events. At that time, the probability of observing rare ACR species was sharply reduced by the combination of an increasing modulation of the ACR intensities and a decreasing percentage of time when we could observe them.

A logarithmic contour plot of the distribution of pulse heights of ions accumulated during all quiet times is shown in Figure 2. On the plot is a superposed energy/nucleon scale for selected values from 3.3 to $20 \mathrm{MeV} / \mathrm{amu}$ for most of the dominant elements. This plot shows the element resolution and its energy variation throughout the study region. A total of 191,938 O ions in the 3.3-20 MeV/amu interval were collected during quiet times in our study period.

Figure 3 shows histograms of the distribution of the deduced atomic number, $Z$, of each ion for three energy intervals. Several characteristics should be noted in these histograms. First, background below $\mathrm{O}$, although at a level of only $\sim 1 \%$ of the $\mathrm{O}$ peak, severely limits our measurement of $\mathrm{C}$, especially at low energies. To correct for this background we have essentially drawn a line between minima above and below $\mathrm{C}$ and counted only the contribution above this line for the $\mathrm{C}$ intensity. A similar correction has been applied to $\mathrm{N}$, but its effect is much smaller. Second, above Ne the presence of elements with odd $\mathrm{Z}$, such as $\mathrm{Na}, \mathrm{Al}, \mathrm{P}$ and $\mathrm{Cl}$ may be seen in the data above $5 \mathrm{MeV} / \mathrm{amu}$. These species are not resolved below $5 \mathrm{MeV} / \mathrm{amu}$ but the dominant species with even $\mathrm{Z}$ are well resolved there. Third, there is no particular evidence of background above $Z=10$ and no reasonable means to determine any small correction that might be necessary; no such correction has been applied. We revisit the question of background in section 4 .

\section{ENERGY SPECTRA AND ABUNDANCES}

Figures 4 and 5 show energy spectra for the dominant elements we are able to study. Figure 4 shows spectra for elements that have a clear ACR component or a low-energy increase while Figure 5 shows species with no statistically convincing evidence of an $\mathrm{ACR}$ component. Note that $\mathrm{Mg}$ and $\mathrm{Si}$ show a clear rise in intensity at low energies while $\mathrm{C}$ and $\mathrm{Fe}$ do not. In Figure 5 we also see a spectrum for the sum of $\mathrm{Ti}$ and $\mathrm{Cr}$, labeled $\mathrm{TiCr}$; the resolution of these elements can be seen in the histograms in Figure 3. These two species, which result from fragmentation of GCR Fe during traversal of interstellar material, show no significant decrease as a fraction of $\mathrm{Fe}$ at low energies. This indicates that the low-energy Fe is consistent with having a GCR origin. In the case of C, while the background correction is large at low energies, the flat spectrum is similar to that of $\mathrm{Fe}$ and the $\mathrm{Fe} / \mathrm{C}$ ratio is also similar to the corresponding GCR ratio.

Abundances of all species near $5 \mathrm{MeV} / \mathrm{amu}$ are shown in Table 1 . This is the lowest energy where we can still estimate abundances of elements such as $\mathrm{Na}, \mathrm{Al}, \mathrm{P}$ and $\mathrm{Cl}$, so we have complete coverage of all listed species. However the low-energy ACR components of $\mathrm{Mg}$ and $\mathrm{Si}$, for example, are not well established at $5 \mathrm{MeV} / \mathrm{amu}$. Note that com- 
paring intensities in the 3.3-4.0 MeV/amu interval, $\mathrm{S}$ exceeds $\mathrm{Fe}$ and the abundances of $\mathrm{Mg}$ and $\mathrm{Si}$ are twice that of Fe.

To remove energy dependence from the abundances and determine abundances or limits for the ACR elements, we must correct for solar modulation. We have made a first-order correction using the force-field approximation (e.g. Fisk 1974), also assuming that all species have the same power-law spectrum at the termination shock and that all are singly ionized. We have adjusted the source-spectral power, $\beta$, the potential $\varphi$, and the normalization for each species to obtain the spectral fits (see Reames et al. 1997a). The optimum parameters are $\beta=-3.0$ and $\varphi=85 \mathrm{MV}$. For elements such as $\mathrm{Mg}, \mathrm{Si}$ and $\mathrm{S}$, the high-energy regions above $\sim 10 \mathrm{MeV} / \mathrm{amu}$, dominated by GCRs, are not included in the fit. For elements such as Fe with no clear ACR contribution, limits are determined from the lowest energy point. The fits are shown in Figure 6 and the corresponding ACR abundances given in the last column of Table 1. Comparing the last 2 columns of Table 1 , it is clear that the derived ACR relative source abundances are extremely insensitive to the details of the modulation correction, with the exception of the abundance of He which changes by a factor of $\sim 3$ between the shock and Earth. Also shown in Table 1 are the "standard abundances" of Grevesse, Noels \& Sauval (1996). These abundances are derived from meteoritic and solar photospheric measurements and are believed to be our best estimate of the abundances in the local region of the galaxy.

Most of the upper limits shown for the ACR abundances in Table 1 are determined because we are unable to distinguish the presence of an ACR component from the GCR background. However, for the heaviest elements, especially $\mathrm{Kr}$, we see no ions at all and we expect no competing GCR component. For $\mathrm{Kr}$, we have seen no ions at any energy, not just near $5 \mathrm{MeV} / \mathrm{amu}$, and we have adjusted the one-particle upper limit accordingly.

Finally, we divide each ACR abundance by the corresponding standard abundance (Grevesse, Noels \& Sauval 1996) shown in Table 1, and plot the result as a function of FIP in Figure 7. Solid circles are used for measured values and open circles for values that are probably only upper limits; error bars on the latter extend to the two standarddeviation points.

It is easy to understand why the relative abundances of $\mathrm{He}$ and $\mathrm{Ne}$ are below those of $\mathrm{N}, \mathrm{O}$, and $\mathrm{Ar}$ in Figure 7 in terms of the relative pickup probabilities for those elements. Photo-ionization cross sections for $\mathrm{He}$ and $\mathrm{Ne}$ are much lower than for the other ions. A full numerical calculation of the ionization probabilities that vary as $R^{-2}$ with distance $R$ from the Sun has been performed by Rucinski et al. (1996).

\section{BACKGROUND}

One of the greatest concerns in the study of rare species at low intensities is the detection and elimination of background. The large-geometry instruments required for this task are inherently more susceptible to multiple particles in a telescope during the electronic response time than are smaller instruments. We automatically eliminate obvious cases, such as simultaneous firing of multiple dome detectors, but we are vulnerable to cases such as a low-energy $O$ stopping in a dome detector at the same time that a proton or He penetrates the detector wall and stops in the PSD. Since $\mathrm{H}, \mathrm{He}$ and $\mathrm{O}$ are by far the 
dominant species at low energies, the greatest background at high pulse heights tends to fall below the track of $O$ in pulse-height space as we can see in Figure 2. However, similar background below $\mathrm{Ne}$ (and above $\mathrm{O}$ ) probably produces the observed limit $\mathrm{F} / \mathrm{Ne}$ $<3 \%$. However, this type of background from Ar cannot significantly affect $S$, since $\mathrm{S} / \mathrm{Ar} \approx 15 \%$ below $5 \mathrm{MeV} / \mathrm{amu}$.

At high energies it is common to design particle telescopes that require 3 or more detector elements in coincidence. This redundancy can be used to eliminate some background caused by multiple particles or by nuclear interactions of GCR protons. However, to retain our energy threshold and geometry we would have had to replace our 48 single 17-micron, $2-\mathrm{cm}^{2}$ detectors with 48 pairs of spatially separated 8.5 -micron detectors of equal or greater area. Such thin large-area detectors are not feasible with current technology. Fortunately, however, the effects of nuclear reactions are greatly reduced with the large-area thin detectors that are used here so the need for multiple measurements to eliminate them is reduced. In short, LEMT was deliberately designed to sacrifice redundancy in order to maximize sensitivity at the lowest possible energy.

Background resulting from inner-heliospheric sources such as SEP- and CIR-related events is also at issue here. It is easy to eliminate the large events by observing their time profiles, but small events are a serious concern. The cumulative effect of small impulsive-flare $\left({ }^{3} \mathrm{He}\right.$-rich) events has been shown to affect quiet-time measurements, especially at solar maximum (Richardson et al. 1990), and persistent low intensities of ions from CIRs have recently been demonstrated (Reames et al. 1997c). However, a largegeometry instrument is also better able to see and eliminate very small events (see e.g. Reames et al. 1997b, 1997c). The lack of a low-energy increase in the Fe spectrum in Figure 5, while puzzling in itself, is evidence that we have eliminated any contribution from Fe-rich impulsive SEP events. On average, impulsive SEP events have Fe/Si $=3.1 \pm 0.3$, gradual events have $\mathrm{Fe} / \mathrm{Si}=0.88 \pm 0.04$ and $\mathrm{CIR}$ events have $\mathrm{Fe} / \mathrm{Si}=0.97 \pm 0.14$ (Reames 1995). Here we measure $\mathrm{Fe} / \mathrm{Si}=0.37 \pm 0.11$ in the $3.3-4.0 \mathrm{MeV} / \mathrm{amu}$ interval; not even one of 49 gradual SEP events (Reames 1995) attained values this low.

\section{DISCUSSION}

Our decision on the presence of an ACR component for most elements has been based primarily on evidence of the presence of an ACR-like spectrum that decreases as an increasing function of energy at low energies. It could be argued that the flat spectra like that of $\mathrm{Fe}$, shown in Figure 5, consist of an ACR component at low energies combined with a GCR spectrum that rises with energy, so as to compensate. We can not completely exclude this possibility, although the high abundances of $\mathrm{Cr}$ and $\mathrm{Ti}$ GCR secondaries argue against it for the case of Fe. This approach would result in changing upper limits to values in Table 1 and in changing open circles to solid circles in Figure 7. We have chosen the more conservative approach that evidence for ACRs must be fairly obvious.

After the reported observation of possible ACR S at 1 AU, ACR components of Si, $\mathrm{S}$, and $\mathrm{Fe}$ (but not $\mathrm{Mg}$ ) were reported from the Voyager spacecraft in the outer heliosphere (Stone \& Cummings 1997). Those authors suggested that the $S$ intensity observed at Wind was about a factor of 3 higher than that expected from the Voyager measurement 
if the radial gradient of $S$ were the same as that of $O$. However, this comparison was made at $8 \mathrm{MeV} / \mathrm{amu}$ where our measurement may contain a GCR contribution and the intensity measurements on the two spacecraft came from different time intervals. We suggest that our value of $\mathrm{S} / \mathrm{Ar}=0.14 \pm 0.05$ derived from Table 1 is well within the large error of the corresponding value measured at Voyager 1 which is $\sim 0.1$ with $\sim 50 \%$ errors.

The presence of $\mathrm{S}^{+}$in the ACRs need not necessarily imply that the source of neutral $S$ is outside the heliosphere. An internal source of $S$ such as S-rich gas from volcanoes on the Jovian moon Io, for example, can not be excluded at present. However, Io is not a likely source of neutral $\mathrm{Mg}$ or $\mathrm{Si}$ and we can not rule out interstellar neutral gas as the origin for all of these species. Once neutrals enter the heliosphere, Si and S have high photoionization cross sections (Zombeck 1992) and both $\mathrm{Mg}$ and Si can be ionized by solar $\mathrm{H} \alpha$ radiation (unlike the high-FIP elements). For $\mathrm{Si}$, the ionization rate is an order of magnitude larger than that for $\mathrm{O}$, therefore an interstellar neutral $\mathrm{Si} / \mathrm{O}$ ratio of $\sim 0.02 \%$ would explain our observation. To some extent, the same physics that reduced the number of interstellar low-FIP neutrals also increases their probability of capture inside the heliosphere.

Our lack of observation of ACR Fe that was seen on Voyager 1 is interesting. At some intensity level, Fe must be accelerated from the tail of the solar wind distribution function by the heliospheric termination shock. This multiply ionized energetic Fe would be more strongly suppressed by modulation than singly ionized $\mathrm{Fe}$, hence it is more likely to be seen on Voyager at $-60 \mathrm{AU}$ than on Wind at $1 \mathrm{AU}$. Actually, $\mathrm{Fe}^{+14}$ in the solar wind has $Q / A=0.25$, the same as that of $\mathrm{He}^{+}$, so both ions should be modulated similarly. However, it is probably premature to draw firm conclusions on the origin of the Fe from early results based on limited statistics.

In principle it should be possible to observe any singly ionized ions of elements such as S on SAMPEX (e.g. Klecker et al 1997). Even above $10 \mathrm{MeV} / \mathrm{amu}$ where most $\mathrm{S}$ ions are from GCRs, the singly ionized ACR ions would be visible in a region of geomagnetic latitude where GCR ions are excluded. Unfortunately, SAMPEX spends a very small fraction of its orbit in this region $(\sim 10 \%)$, so it is extremely unlikely that the instruments on SAMPEX can see any $\mathrm{S}^{+}$or other rare ACR ions, even with a geometry factor that is otherwise larger than that of LEMT. For example, an instrument with a geometry factor of $60 \mathrm{~cm}^{2}$ sr would have an effective geometry factor of only $\sim 6 \mathrm{~cm}^{2} \mathrm{sr}$ for $\mathrm{S}^{+}$.

We have made an effort to extend our study to $\mathrm{Kr}$ because it is the only remaining element with a FIP greater than that of $\mathrm{H}$ that has not yet been observed in the ACRs. Such elements are important in defining the way the pickup process depends upon FIP and in distinguishing differences in pickup efficiencies from differences in interstellar abundances. Unfortunately, our limit is still a factor of 10 greater than the expected value. It is unlikely that this situation will be improved soon since contemporary instruments other than LEMT are unable to observe elements with $\mathrm{Z}>30$.

\section{CONCLUSIONS}

We have reported the energy spectra and abundances or upper limits for 21 elements from He through $\mathrm{Kr}$ during quiet periods of solar minimum between 1994 November and 
1998 April. Results indicate that we have successfully removed any contribution of impulsive or gradual SEP events or ions accelerated at CIRs from the measurements. The elements, $\mathrm{He}, \mathrm{N}, \mathrm{O}, \mathrm{Ne}, \mathrm{Mg}, \mathrm{Si}, \mathrm{S}$, and Ar show evidence of an ACR component. The ACR abundances of $\mathrm{He}, \mathrm{N}, \mathrm{O}, \mathrm{Ne}$, and $\mathrm{Ar}$ are in good agreement with the values expected from the pickup and acceleration of interstellar neutrals with abundances similar to the standard photospheric abundances.

For Fe, there is no clear evidence of a spectral increase at low energies and $\mathrm{Ti}$ and $\mathrm{Cr}$ are consistent with the abundances expected from fragmentation of GCR Fe, not with the abundances expected in solar, meteoritic or local galactic material. The backgroundcorrected spectrum and abundance of $C$ is also consistent with a GCR origin, as is the $\mathrm{Fe} / \mathrm{C}$ ratio. However, instruments with better background rejection than LEMT might be better able to resolve an ACR component of $\mathrm{C}$ from the background at a level of $\sim 1 \%$ of O. Mewaldt et al. (1996) report an ACR component of $\mathrm{C}$ with $\mathrm{C} / \mathrm{O}=0.014 \pm 0.009$ from SAMPEX and $0.020 \pm 0.004$ from Voyager.

Interesting differences exist between the spectral and abundance observations of $\mathrm{Mg}$, $\mathrm{Si}$, and $\mathrm{Fe}$ at Voyager and at Wind. However, attempts to explain those differences, and to identify the origin of the particles, are somewhat speculative and are probably premature at present level of statistical accuracy of the measurements.

Finally, our attempt to measure the abundance of $\mathrm{Kr}$, the last high-FIP element in the ACRs, results in $\mathrm{Kr} / \mathrm{O}<0.02$. This limit is a factor of 10 larger than the expected ratio.

The author thanks T. T. von Rosenvinge for his support and assistance during the course of this work. 


\section{REFERENCES}

Adams, J. H., et al. 1991, ApJ 375, L45.

Cummings, A. C., \& Stone, E. C. 1996, Space Sci. Revs., 78, 117.

Fisk, L. A. 1974, in High Energy Particles and Quanta in Astrophysics, edited by F. B. McDonald and C. E. Fichtel (Cambridge Mass: MIT Press), p. 170.

Fisk, L. A. 1996, Space Sci. Revs., 78, 129.

Fisk, L.A., Kozlovsky, B., \& Ramaty, R. 1974, ApJ 190, L35.

Geiss, J., Gloeckler, G., Mall, U., von Steiger, R., Galvin, A. B., and Ogilvie, K., 1994, A\&A 282, 924.

Gloeckler, G., et al. 1993, Science 261, 70.

Grevesse, N., Noels, A., and Sauval, A. J., 1996, in Cosmic Abundances, edited by S. Holt and G. Sonneborn, A. S. P. Conf. Series 99, p. 117.

Hasebe, N., et al. 1997, Adv. Space Res., 19, No. 5, 813.

Klecker, B. 1995, Space Sci. Revs. 72, 419.

Klecker, B., Mewaldt, R. A., Oetlicker, M., Selesnick, R. S., and Jokipii, J. R. 1998, Space Sci. Revs. 83, 294.

Klecker, B., Oetliker, M., Blake, J. B., Hovestadt, D., Mason, G. M., Mazur, J. E., \& McNab, M. C. 1997, Proc 25 ${ }^{\text {th }}$ Int. Cosmic Ray Conf (Durban), 273.

Lee, M. A. 1996, Space Sci. Revs., 78, 109.

Lund, N. 1989, in Cosmic Abundances of Matter, sdited by C. J. Waddington A. I. P. Conf. Proc. 183, p. 111.

McDonald, F. B., Teegarden, B. J., Trainor, J. H., \& Webber, W. R. 1974, ApJ 187, L105.

Meyer, J. P. 1985, ApJS 57, 151.

Meyer, J. P. 1989, in Cosmic Abundances of Matter, sdited by C. J. Waddington A. I. P. Conf. Proc. 183, p. 245.

Mewaldt, R. A., Cummings, J. R., Leske, R. A., Selesnick, R. S., and Stone, E. C. 1996, GRL 23, 617.

Mewaldt, R. A., Selesnick, R. S., Cummings, J. R., Stone, E. C., and von Rosenvinge, T. T., 1996, ApJ 466, L43.

Möbius, E., Hovestadt, D., Klecker, B., Scholer, M., Gloeckler, G., \& Ipavich, F. M. 1985, Nature 318, 426.

Pesses, M. E., Jokipii, J. R., \& Eichler, D. 1981, ApJ 246, L85.

Reames, D. V. 1995, Adv. Space Res., $15(7), 41$.

Reames, D. V., Barbier, L. M., \& von Rosenvinge, T. T., 1997a, Adv. Space Res., 19 (5), 809. 
Reames, D. V., Barbier, L. M., von Rosenvinge, T. T., Mason, G. M., Mazur, J. E., \& Dwyer, J. R. 1997b, ApJ, 483, 515.

Reames, D, V., Ng, C. K., Mason, G. M., Dwyer, J. R., Mazur, J. E., \& von Rosenvinge, T. T. 1997c, Geophys. Res. Letters 24, 2917.

Richardson, I. G., Reames, D. V., Wenzel, K. P. \& Rodriguez-Pacheco, J. 1990, ApJ. 363, L9.

Rucinski, D., Cummings, A. C., Gloeckler, G., Lazarus, A. J., Möbius, E., and Witte, M. 1996, Space Sci. Revs., 78, 73.

Stone, E. C., \& Cummings, A. C. 1997, Proc 25 ${ }^{\text {th }}$ Int. Cosmic Ray Conf (Durban), 2, 289.

Takashima, T., et al. 1997, ApJ 477, L111.

Zombeck, M. V. 1992, Handbook of Space Astronomy and Astrophysics (Cambridge Univ. Press), p 198. 


\section{FIGURE CAPTIONS}

Fig. 1. The percentage of time during each 27-day solar rotation period that is quiet and 27-day averaged intensities of $\mathrm{O}$ are shown as a function of time during the study period.

Fig. 2. The distribution of pulse heights in the dome detector vs. the corresponding pulse height in the position-sensing energy detector is shown for all ions entering the LEMT telescope during quiet times.

Fig. 3. Histograms of the distribution of the deduced atomic number, $Z$, of each ion are shown for the three energy intervals indicated.

Fig 4. Energy spectra of ions are shown for species that seem to have an ACR component.

Fig 5. Energy spectra of ions are shown for species that do not show clear evidence of an ACR component.

Fig 6. Fitted spectra are shown together with the observations for representative species with an ACR component. Fits are derived assuming power-law source spectra, singly ionized elements and force-field modulation. $\mathrm{Mg}$ has been omitted from the figure for clarity; its spectrum is similar to that of $\mathrm{Si}$.

Fig. 7. Deduced ACR abundances at the acceleration site divided by the corresponding standard (local-galactic) abundances are plotted as a function of the FIP of the element. Elements with clear ACR components are shown as solid circles with heavy error bars, limiting values are shown as open circles. 
Table 1. Quiet-Time Element Abundances

\begin{tabular}{cccccc}
\hline & $\mathrm{Z}$ & $\mathrm{FIP}$ & $\begin{array}{c}\text { Standard } \\
\text { Abundance }\end{array}$ & $\begin{array}{c}\sim 5 \mathrm{MeV} / \mathrm{amu} \\
\text { Quiet Time }\end{array}$ & $\begin{array}{c}\text { Anomalous } \\
\text { Component }\end{array}$ \\
\hline $\mathrm{He}$ & 2 & 24.46 & $132000 \pm 11000$ & $1612 \pm 8$ & $5000 \pm 500$ \\
$\mathrm{C}$ & 6 & 11.22 & $479 \pm 55$ & $7 \pm 7$ & $<10$ \\
$\mathrm{~N}$ & 7 & 14.48 & $126 \pm 20$ & $139 \pm 2$ & $120 \pm 10$ \\
$\mathrm{O}$ & 8 & 13.55 & $1000 \pm 161$ & $1000 \pm 6$ & $1000 \pm 10$ \\
$\mathrm{~F}$ & 9 & 17.34 & $0.05 \pm 0.03$ & $<2$ & $<2$ \\
$\mathrm{Ne}$ & 10 & 21.47 & $162 \pm 22$ & $74 \pm 2$ & $70 \pm 7$ \\
$\mathrm{Na}$ & 11 & 5.12 & $2.9 \pm 0.2$ & $\sim 0.2$ & $<0.2$ \\
$\mathrm{Mg}$ & 12 & 7.61 & $51 \pm 6$ & $1.0 \pm 0.2$ & $1.2 \pm 0.3$ \\
$\mathrm{Al}$ & 13 & 5.96 & $4.0 \pm 0.6$ & $\sim 0.1$ & $<0.1$ \\
$\mathrm{Si}$ & 14 & 8.12 & $48 \pm 5$ & $1.4 \pm 0.2$ & $1.7 \pm 0.3$ \\
$\mathrm{P}$ & 15 & 10.9 & $0.38 \pm 0.04$ & $\sim 0.1$ & $<0.1$ \\
$\mathrm{~S}$ & 16 & 10.3 & $29 \pm 7$ & $0.47 \pm 0.14$ & $0.6 \pm 0.2$ \\
$\mathrm{Cl}$ & 17 & 12.95 & $0.4 \pm 0.3$ & $\sim 0.1$ & $<0.1$ \\
$\mathrm{Ar}$ & 18 & 15.68 & $4.5 \pm 1.0$ & $3.7 \pm 0.4$ & $4.2 \pm 0.5$ \\
$\mathrm{Ca}$ & 20 & 6.09 & $3.09 \pm 0.14$ & $0.08 \pm 0.05$ & $<0.1$ \\
$\mathrm{Ti}$ & 22 & 6.81 & $0.14 \pm 0.02$ & $0.12 \pm 0.07$ & $<0.1$ \\
$\mathrm{Cr}$ & 24 & 6.74 & $0.63 \pm 0.04$ & $\sim 0.1$ & $<0.1$ \\
$\mathrm{Fe}$ & 26 & 7.83 & $42.7 \pm 3.9$ & $0.8 \pm 0.2$ & $<0.9$ \\
$\mathrm{Ni}$ & 28 & 7.61 & $2.4 \pm 0.05$ & $\sim 0.04$ & $<0.05$ \\
$\mathrm{Zn}$ & 30 & 9.36 & $0.054 \pm 0.010$ & $<0.04$ & $<0.05$ \\
$\mathrm{Kr}$ & 36 & 13.93 & $0.0023 \pm 0.0001$ & $<0.02$ & $<0.02$ \\
\hline & & & & &
\end{tabular}



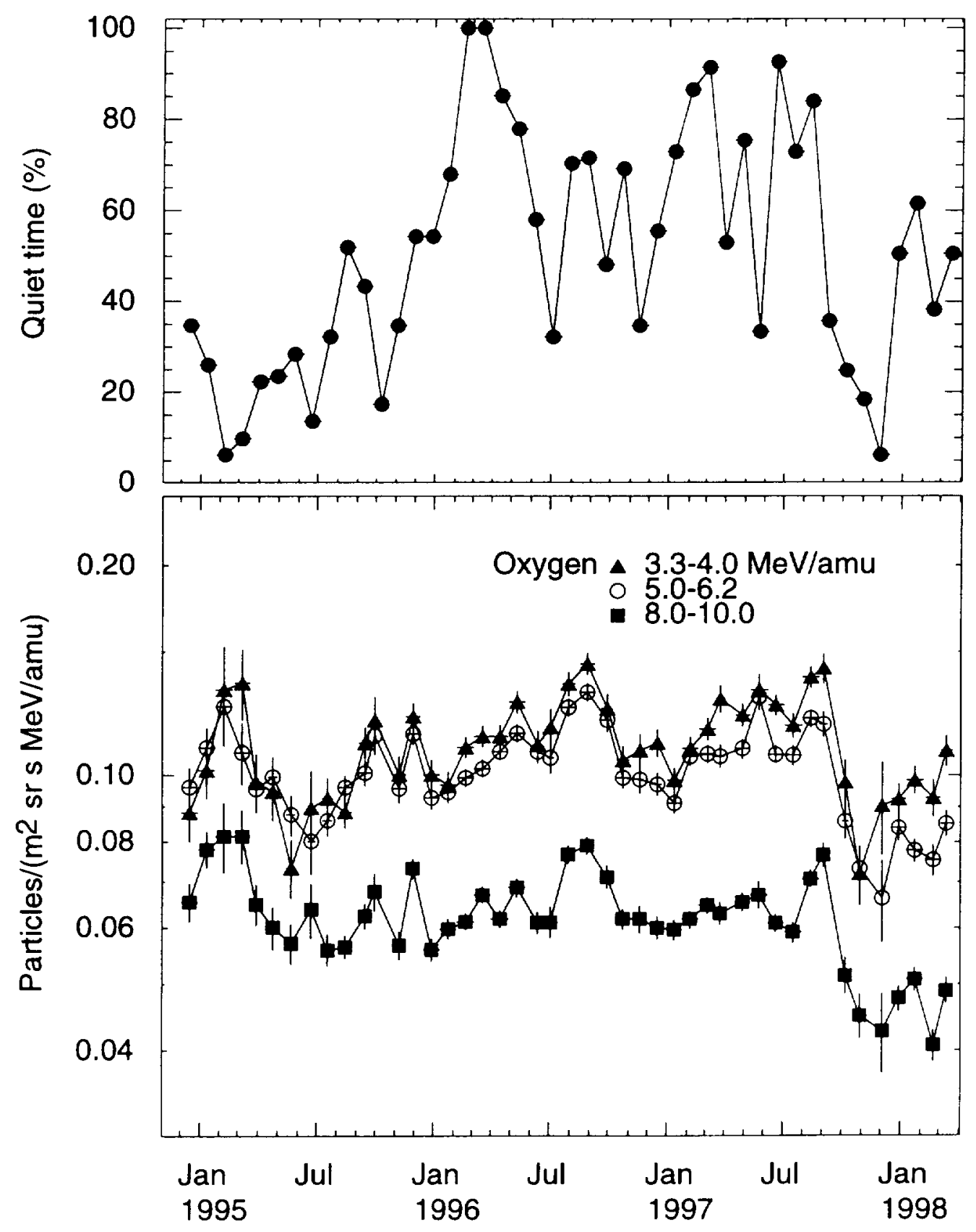

Fig. 1 


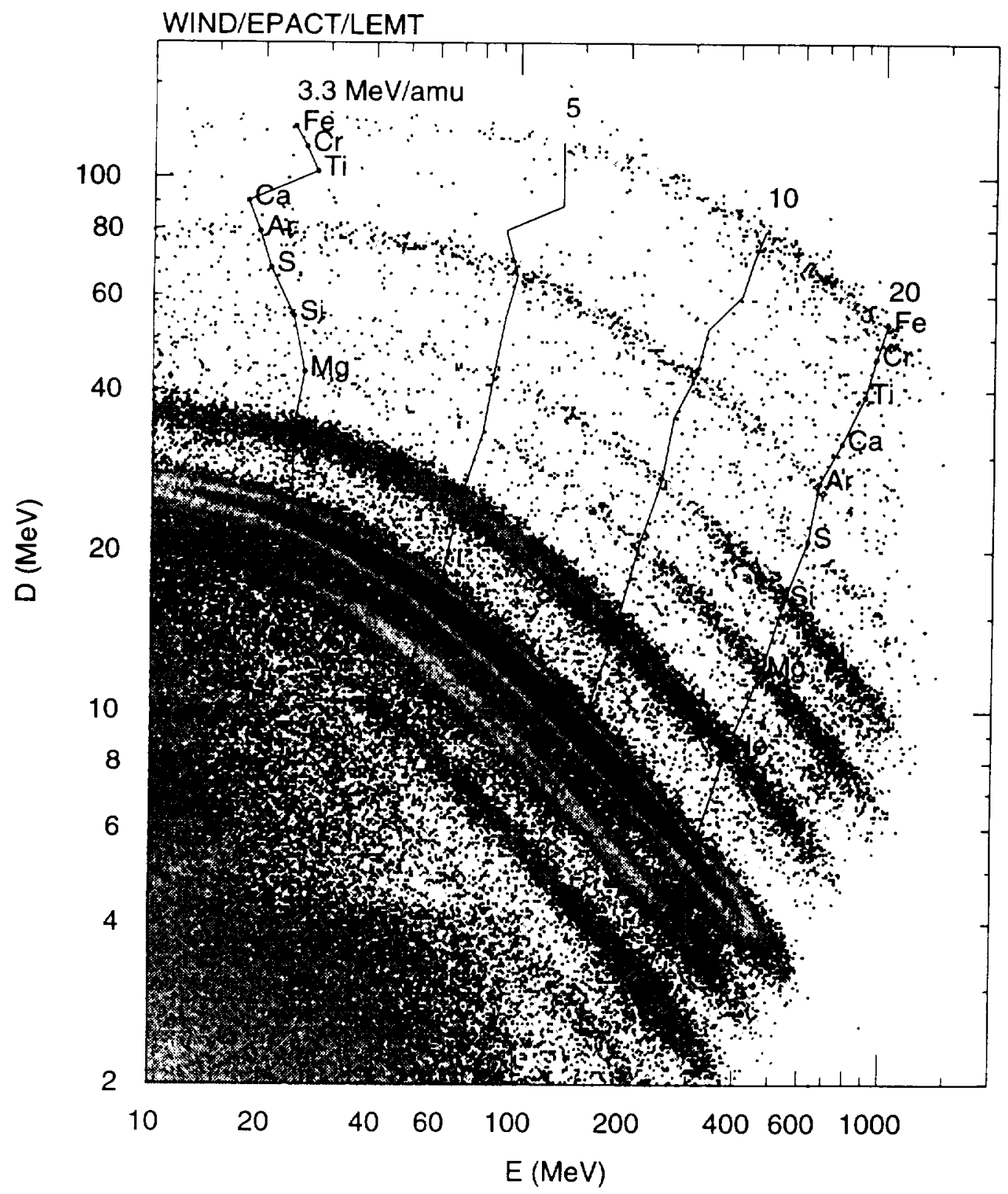




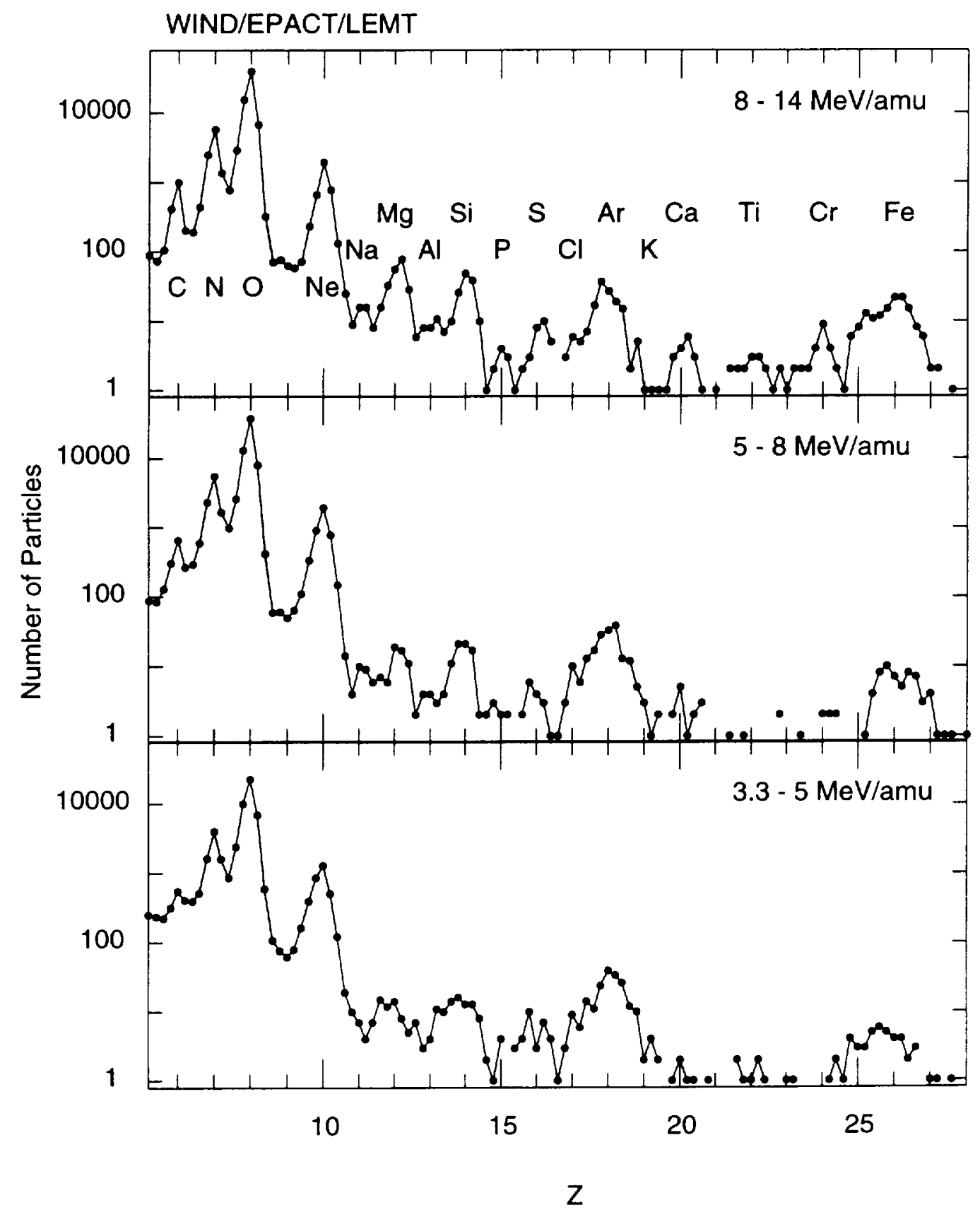

Fig. 3 


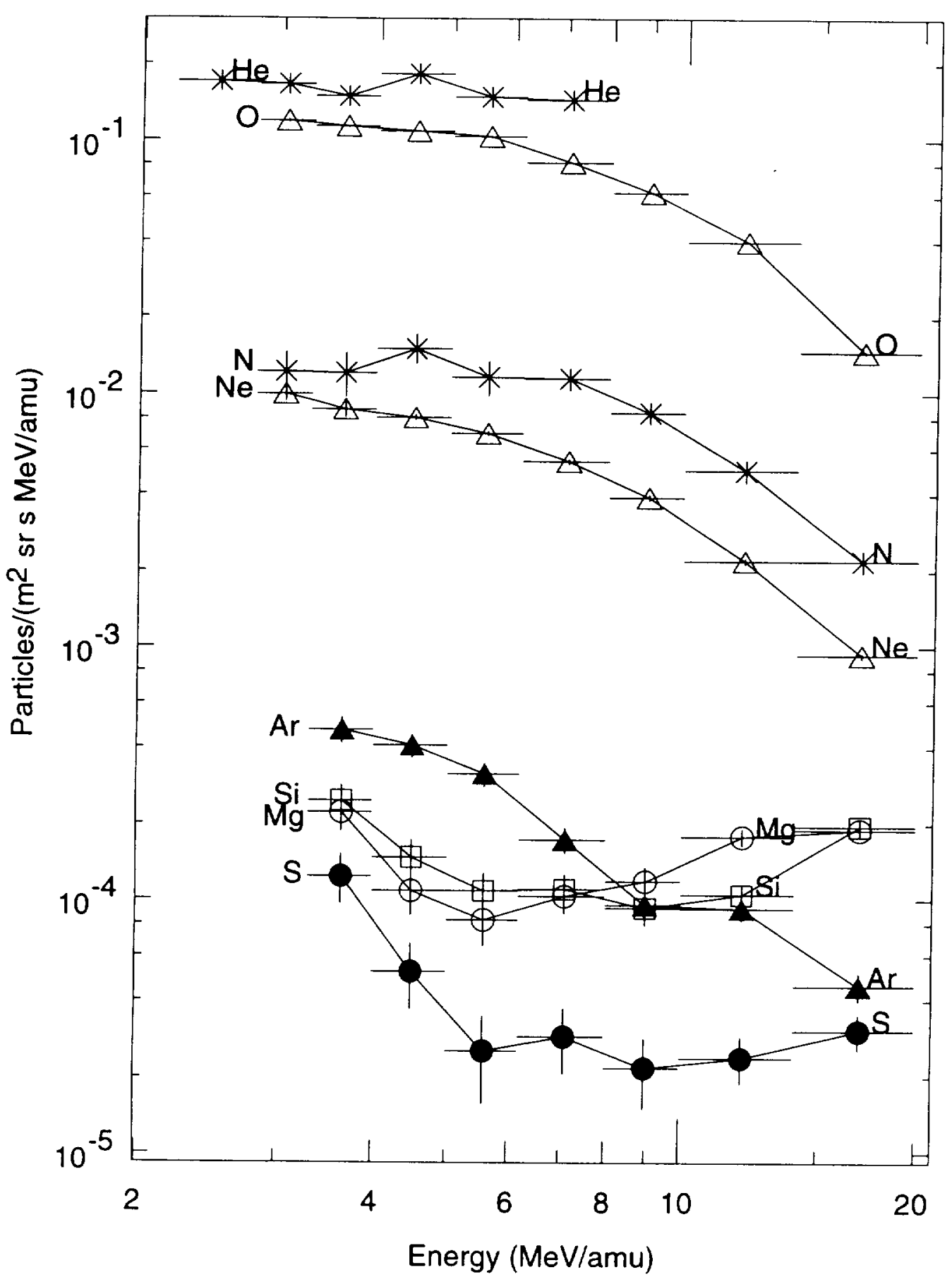

Fig. 4 


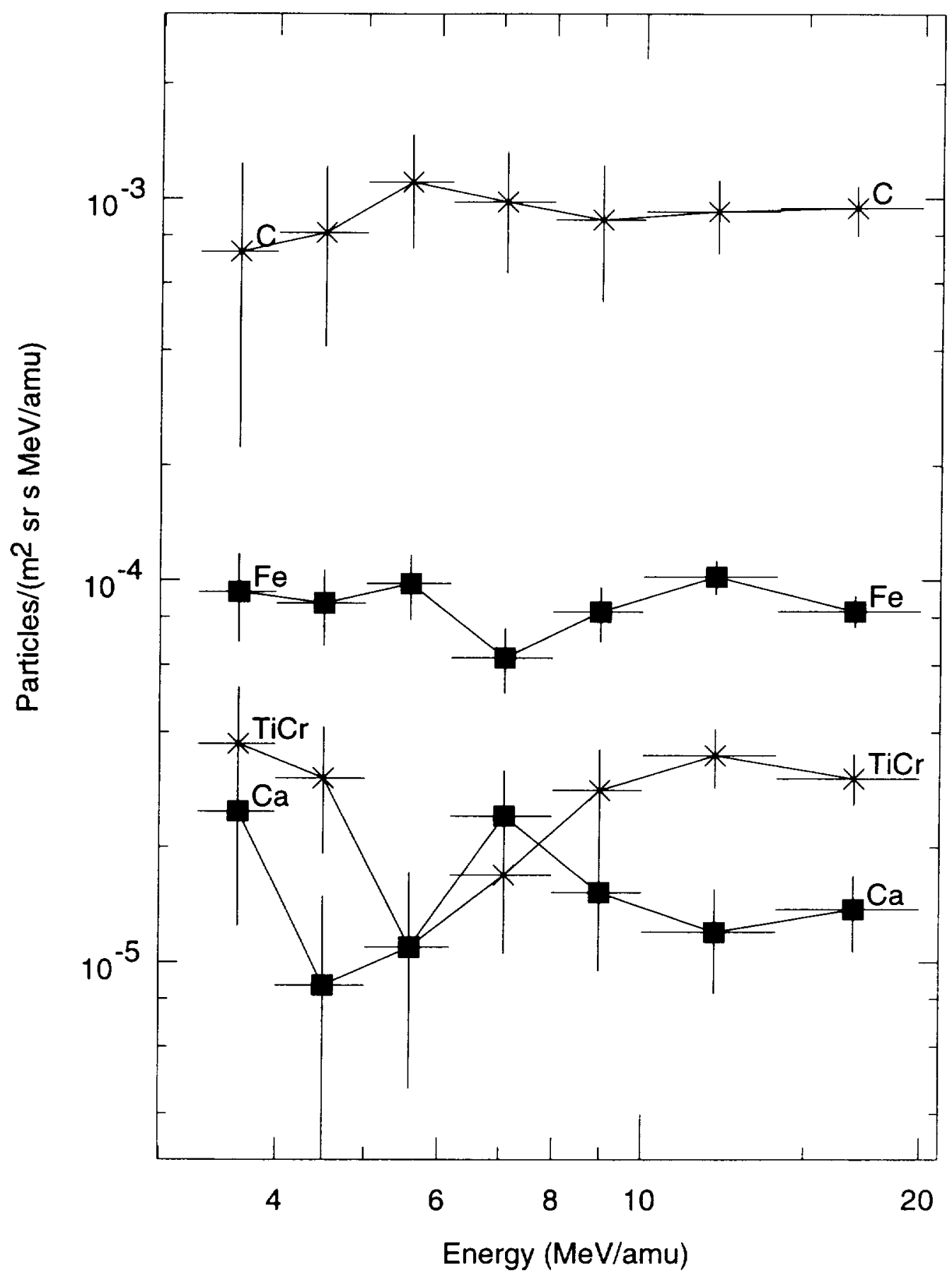

Fig. 5 


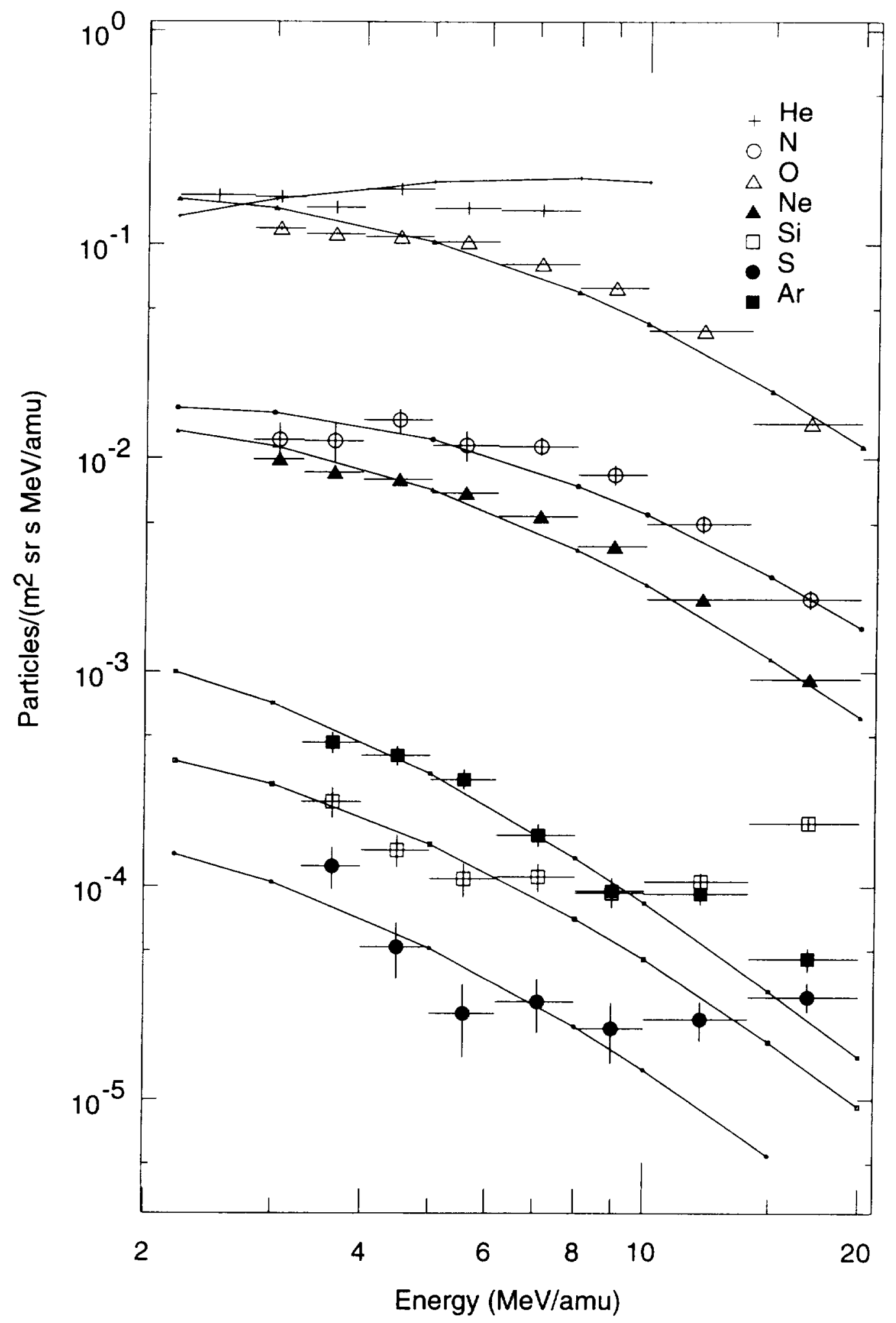

Fig. 6 


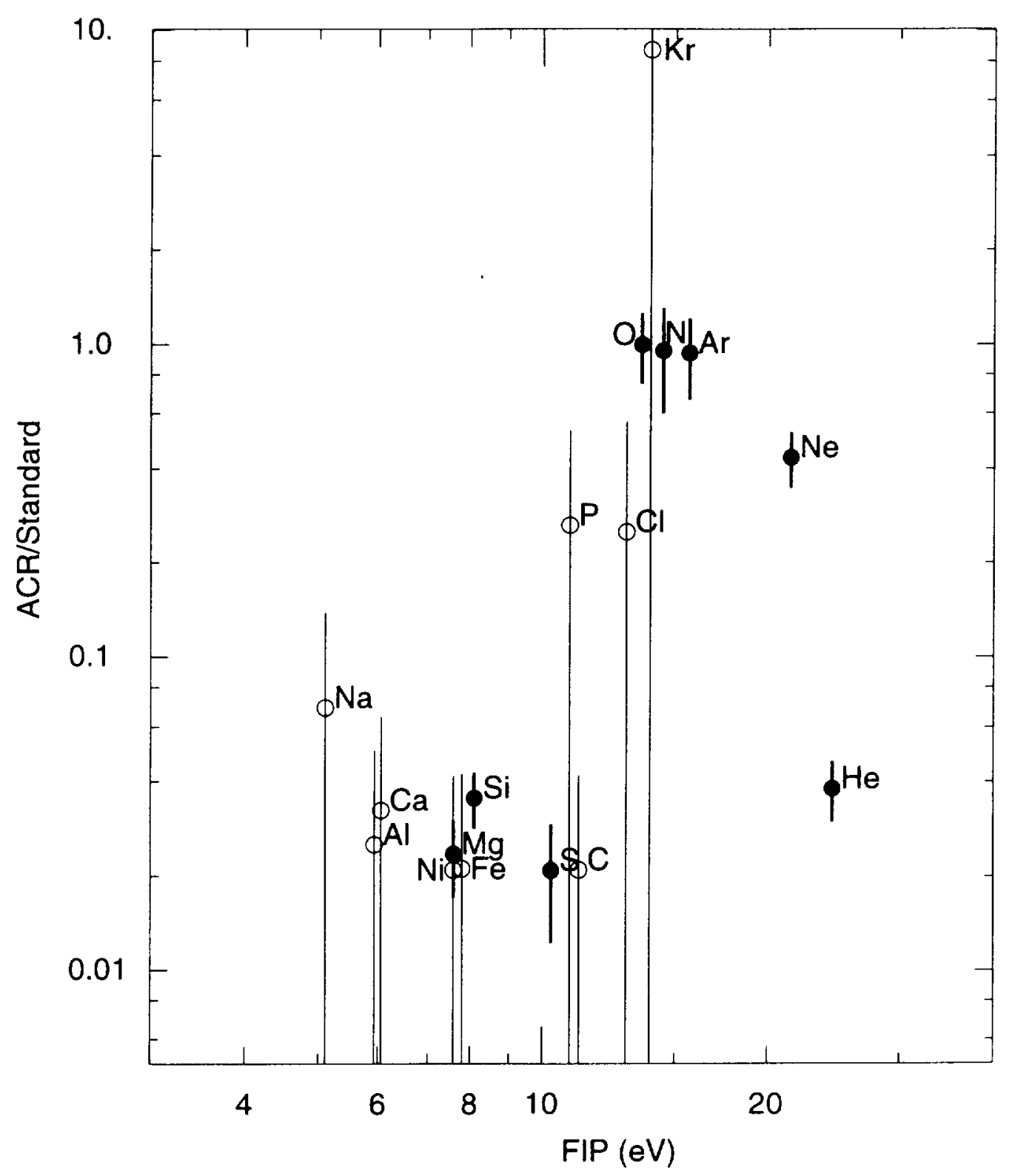

Fig. 7 